\title{
LA CAPACIDAD DE CARGA PSICOSOCIAL DEL TURISTA: INSTRUMENTO DE MEDICIÓN PARA EL DESARROLLO SOSTENIBLE EN LA TURISTIFICACIÓN DE LOS CENOTES ${ }^{1}$
}

\author{
Rocío Blanco Gregory* \\ Universidad de Extremadura \\ Fernando Enseñat Soberanis** \\ Universidad de Quintana Roo - Universidad Autónoma de Yucatán \\ Johnathan Alexander Mondragón Mejía*** \\ Universidad Nacional Autónoma de México
}

\section{RESUMEN}

La capacidad de carga psicosocial ha demostrado ser un instrumento efectivo para establecer números máximos de personas que un turista está dispuesto a ver simultáneamente en espacios naturales. Aunque, ha sido muy aplicado en parques nacionales de EEUU, este indicador no ha sido utilizado en espacios naturales confinados como son los cenotes de la península de Yucatán. Esta investigación aplica este indicador generando resultados que pueden utilizarse para una gestión sostenible de estos espacios.

Palabras clave: Percepción de multitud, Turismo sostenible, Capacidad de carga psicosocial, Turistificación, Cenote.

Fecha de recepción: 4 de diciembre de 2017

Fecha de aceptación: 15 de mayo de de 2018

* Departamento de Dirección de Empresas y Sociología. Facultad de Empresa, Finanzas y Turismo. Universidad de Extremadura. Avda. de la Universidad, s/n. 10076 CÁCERES (España). E-mail: rblanco@unex.es

**Doctorado en Desarrollo Sostenible. División de Desarrollo Sustentable (DSS). Universidad de Quintana Roo. Avenida Andrés Quintana Roo s/n, Colonia San Gervasio. 77600 COZUMEL, Quintana Roo (México). Facultad de Ciencias Antropológicas, Universidad Autónoma de Yucatán, Km 1, carr. Mérida- Tizimín, MÉRIDA, Yucatán (México) E-mail:fernandoensenat@gmail.com

***Unidad Multidisciplinaria de Docencia e Investigación. Facultad de Ciencias. Universidad Nacional Autónoma de México (Campus Sisal). Puerto de Abrigo, s/n. 97356 SISAL, Yucatán (México). E mail: johnybc123@gmail.com1

Agradecimientos: Al Consejo Nacional de Ciencia y Tecnología (CONACyT). CVU: 339845. 


\title{
The psychosocial load capacity of the tourist: instrument of measurement for the sus- tainable development in the touristification of the cenotes
}

\begin{abstract}
The psychosocial load capacity has proven to be an effective instrument to establish maximum numbers of people that a tourist is willing to see simultaneously in natural spaces. Although, it has been widely applied in national parks in the USA, this indicator has not been used in confined natural spaces such as the cenotes of the Yucatan Peninsula. This research applies this indicator generating results that can be used for sustainable management of these spaces.
\end{abstract}

Keywords: Perception of crowd, Sustainable Tourism, Psychosocial load capacity, Tourist massification, Cenote.

\section{INTRODUCCIÓN}

Actualmente el turismo, en todas sus modalidades, es una de las industrias con mayor crecimiento. Representa una actividad importante en el desarrollo económico de los países al generar divisas. Hemos de partir de la base de que, según fuentes consultadas de SECTUR en 2016, el turismo en el año 2015, a pesar de crecer a nivel mundial, en México duplicó este crecimiento con una tasa del 9,5\%. Se trata de la industria que ha sufrido el crecimiento más rápido en el siglo XX. Y éste es un hecho que en México se ha desarrollado aún más. Evidentemente, esta situación consigue fuertes inyecciones de dinero para las zonas de destino, pero, a la vez, se corre el riesgo de que una gestión inadecuada haga que ciertas actividades turísticas se masifiquen demasiado, lo cual tendría implicaciones negativas en el mantenimiento y conservación de la naturaleza y del medio ambiente, así como en la experiencia del visitante, hechos que afectarían a la continuidad de esas prácticas turísticas en los cenotes $^{2}$ de la península de Yucatán, como es el caso que aquí se está presentando.

De igual forma, el turismo trae consigo implicaciones sociales al incidir en el crecimiento urbano, tomando en cuenta patrones de ocupación espacial, e influir en los procesos de conservación ambiental (Schéou, 2009; Marín, García y Daltabuit, 2012).

En los países en vías de desarrollo, el turismo en espacios naturales es considerado como una estrategia económica que contribuye a la conservación y la generación de ingresos para las comunidades rurales (Drumm y Moore, 2002). Para el caso de la Península de Yucatán, al igual que para el resto de las regiones con potencial de turismo de naturaleza

2 Los cenotes son cuevas inundadas con agua dulce que se han formado en circunstancias muy especiales: el lecho es de piedra caliza y el lago se nutre de aguas subterráneas. Aunque los más famosos son los mencionados de México, también hay en otros lugares. El término "cenote" proviene de la palabra maya "dzonot" o "ts'onot", que significa pozo sagrado o abismo. Para esta cultura, representaban la principal fuente de agua dulce en una región que no cuenta lagos y sufre largas temporadas de sequías. Actualmente, una gran mayoría de estos cenotes de la Península de Yucatán se han convertido en un importante atractivo turístico. 
en el país, los pobladores rurales han subsistido con el uso de diversas actividades económicas como son: la agricultura, la ganadería, la caza, la pesca, el aprovechamiento de productos maderables y no maderables, entre otros; sin embargo, el proceso de modernización agrícola y el surgimiento de la actividad turística en la región, afectaron a los precios de los productos agropecuarios y a la participación de los campesinos en la economía nacional dando pauta a la búsqueda de alternativas complementarias de ingreso (Córdoba, 1984). En el caso del cenote Dzombakal, localizado en el Ejido de San Antonio Mulix, en el estado mexicano de Yucatán, sus habitantes decidieron acondicionar dos cenotes para un uso turístico-recreativo y complementar así, sus ingresos provenientes en su mayoría de actividades primarias.

En la actualidad, el turismo forma parte de las actividades que muchas comunidades rurales de la zona maya han abrazado debido a que les proporciona empleos e ingresos. Aunado al auge que ha tenido esta cultura y la promoción del simbolismo espiritual que representan los cuerpos de agua de la región (Martos, 2007), los cenotes se han vuelto parte importante de la oferta de turismo de naturaleza de la península y un foco de atención para dichas comunidades rurales que dependen de ellos, antes para realizar actividades meramente agropecuarias, ahora para un aprovechamiento turístico-recreativo. Este creciente interés está ocasionando que algunos cenotes vean un aumento exponencial en su número de visitantes con los impactos positivos y negativos que este crecimiento trae consigo.

El presente trabajo propone una herramienta de planificación del turismo en cenotes masificados a través de la estimación de la capacidad de carga psicosocial (perceptual del visitante en un contexto social) que use como indicador el nivel de saturación de visitantes o turistas al mismo tiempo en un cenote.

\section{CAPACIDAD DE CARGA PERCEPTUAL}

Las primeras aplicaciones científicas de la capacidad de carga se dieron en la pesca y la ganadería en las décadas de 1920 y 1930, principalmente en el manejo de ranchos y vida silvestre. No fue hasta la década de 1960 cuando se aplicó por vez primera en parques naturales y espacios al aire libre, buscando estimar el uso máximo que un área natural debería tener antes de que el recurso comenzara a dañarse de manera inaceptable (Manning y Anderson, 2012).

Esta idea de "limitar" la frecuencia de visitantes bajo el argumento de daño al patrimonio genera aún mucha controversia debido a la poca evidencia científica que demuestre que, limitando el número de visitantes en un sitio, el daño disminuirá. "Establecer un punto de saturación de manera científica no es posible y más bien responde a una tentativa de los gestores de grandes sitios patrimoniales a usar criterios científicos improbables para legitimar sus decisiones políticas, en lugar de gestionar de otra manera los conflictos de acceso al sitio [...]. No se trata ni del patrimonio, ni del turismo, sino del ejercicio del poder. Poder para hacernos visitar un sitio patrimonial de la forma como ellos [los gestores] quieren que lo visitemos" (Équipe MIT, 2002:281). Así, aunque pareciera que no hay consenso sobre la importancia de calcular o no la capacidad de carga, lo cierto es que la literatura coincide en que, si bien no es un "número mágico" que solucionará 
los problemas de visita masiva y daño al patrimonio, sí representa una línea de base para una adecuada planificación y manejo de los espacios naturales. En este sentido, como argumentan Tudela Serrano y Giménez Alarte (2008), el concepto de capacidad de carga no debe contemplarse como una fórmula matemática o como un método mecánico de determinar los límites óptimos de crecimiento del desarrollo turístico o de identificar los impactos de la actividad turística.

Si bien en sus orígenes, la capacidad de carga buscaba establecer límites en el número de visitantes y minimizar así sus impactos al medio ambiente, con el tiempo se volvió evidente que otros aspectos, como la experiencia del visitante, deberían también ser tomados en cuenta. Coccossis (2013) propone la definición de capacidad de carga del visitante como la estimación del número máximo de personas que pueden estar en un sitio sin que su presencia cause daños irreversibles en el ambiente construido y natural, y se produzca una disminución en la calidad de su experiencia. La capacidad de carga de visitantes tiene, a su vez, tres dimensiones: la física, la perceptual y la administrativa (García Hernández, de la Calle Vaquero y Mínguez García, 2011). La capacidad de carga física (CCF) se refiere al número máximo de personas que la superficie de un sitio puede recibir tomando en cuenta la superficie en metros o kilómetros cuadrados. La capacidad de carga perceptual (CCP) estima el número máximo de individuos que un turista está dispuesto a ver en un área determinada antes de que su nivel de satisfacción comience a decrecer. Por último, la capacidad de carga administrativa (CCA) se refiere al número de visitantes máximo que el personal de un sitio es capaz de manejar de acuerdo a los recursos económicos y humanos con los que cuenta.

El presente trabajo se focaliza en la capacidad de carga perceptual (CCP), que en muchas ocasiones suele asociarse con la capacidad de carga psicosocial del turista o visitante. La diferencia es que la primera está comprendida dentro de la segunda. En efecto, la capacidad de carga psicosocial es un término más amplio que considera a la capacidad de carga perceptual además de otros conceptos, como el nivel de conflicto entre turistas y habitantes locales reflejado en el número máximo de turistas que un habitante local espera ver en el sitio; nivel de conflicto por diferentes usos que se le puede dar a un mismo espacio natural (ej. ciclistas y corredores que usan un mismo sendero dentro de un Parque Nacional); y diferentes motivaciones en función del tipo de turista (Burns, Arnberger y Von Ruschkowski, 2010).

La capacidad de carga perceptual usa como indicador, el nivel de multitud o crowding para medir la aceptabilidad del turista a ver determinado número de personas al mismo tiempo en un espacio natural. Este nivel de multitud refleja estándares o números máximos aceptables de visitantes y su variabilidad dependerá, en gran medida, de las normas personales y sociales de cada turista y de cada grupo social.

\subsection{Teoría Normativa}

Desarrolladas en los campos de la sociología y la psicología social, las normas han atraído considerable atención como construcción teórica y marco empírico en la investigación y la gestión de la recreación al aire libre. En particular, la teoría normativa tiene 
una aplicación especial para establecer estándares de multitud o saturación de visitantes en sitios recreativos.

Al aplicarse las normas en la recreación al aire libre, estas suelen ser definidas como estándares que los individuos y los grupos usan para evaluar el comportamiento y las condiciones sociales y ambientales. Así lo han evidenciado en sus trabajos otros autores como Donnelly, Vaske y Shelby (1992); Shelby y Vaske (1991); Vaske, Graefe, Shelby y Heberlein (1986). En esta misma línea, Manning et al. (1999) apuntan que, si los visitantes tienen normas relativas a los aspectos de las experiencias recreativas, esas normas pueden medirse y utilizarse como base para formular normas de calidad. De esta manera, la capacidad de carga perceptual se puede determinar y gestionar de manera más eficaz. Al respecto, hay que añadir el conflicto que se desprende de las normas sociales y las consecutivas sanciones sociales que pueden conllevar la no observación de dichas normas, pues las normas sociales pueden ser efectivas a nivel social y no tanto a nivel individual de las personas que visitan y hacen uso de las áreas recreativas, o, por el contrario, pueden ser más efectivas a la hora de regular el comportamiento individual y no el colectivo.

Hay que señalar que, generalmente, las normas se refieren a lo que se considera normal o aceptado dentro de un grupo social. Dichas normas representan un conjunto de reglamentos y directrices que especifican cómo se espera que las personas se comporten bajo unas condiciones particulares.

Siguiendo a Vaske y Whittaker (2004), en psicología social, las normas se miden y se aplican fundamentalmente a través de tres paradigmas: los enfoques de normas y modelos de activación, la teoría de la acción razonada y los modelos de características estructurales. Éstos últimos se utilizan ampliamente en la formulación de normas en parques y áreas afines, y tienen como objetivo comprender las características de las normas sociales usando el Modelo de Retorno Potencial (RPM) desarrollado por Jackson en 1965. Según Jackson (1966:36), citado por Alazaizeh et al. (2015), el RPM mide la relación existente entre comportamiento y aprobación dentro de un grupo social; "Representa una definición de la norma en términos de la distribución de la aprobación y la desaprobación potencial por los demás para diferentes opciones de comportamiento mostradas por los actores a lo largo de un continuo, bajo condiciones especificadas, es decir, en una determinada situación definida”.

Usando esta metodología, las normas personales de los turistas pueden ser agregadas para probar la existencia de normas sociales o el grado en que las normas son compartidas entre grupos. La investigación normativa en recreación al aire libre se ha centrado en gran medida en el problema de la aglomeración o saturación de visitantes (Vaske y Donnelly, 1988, Shelby, Vaske y Harris, 1988).

El Modelo de Retorno Potencial propuesto por Jackson (1965, 1966) (ver Fig. 1) incluye dos componentes principales: la dimensión de comportamiento (eje X) que representa un número de comportamientos posibles de condiciones de un indicador en una situación particular y la dimensión de evaluación (eje Y) (por ejemplo, aceptabilidad). La curva de potencial de retorno, también denominada con frecuencia curva de normas sociales, se representa para describir los sentimientos de los miembros del grupo sobre una dimensión específica del comportamiento en una situación concreta. Las calificaciones de actitud de los miembros individuales del grupo se promedian y sirven como base para 
la curva. Se pueden cuantificar varias características de la curva para medir y describir diferentes características de las normas, tales como el intervalo de condición aceptable, la intensidad de la norma, la condición óptima o preferida, la condición mínima aceptable y la cristalización de la norma (Alazaizeh et al., 2015).

\section{Figura 1}

\section{CURVA DE LA NORMA SOCIAL}

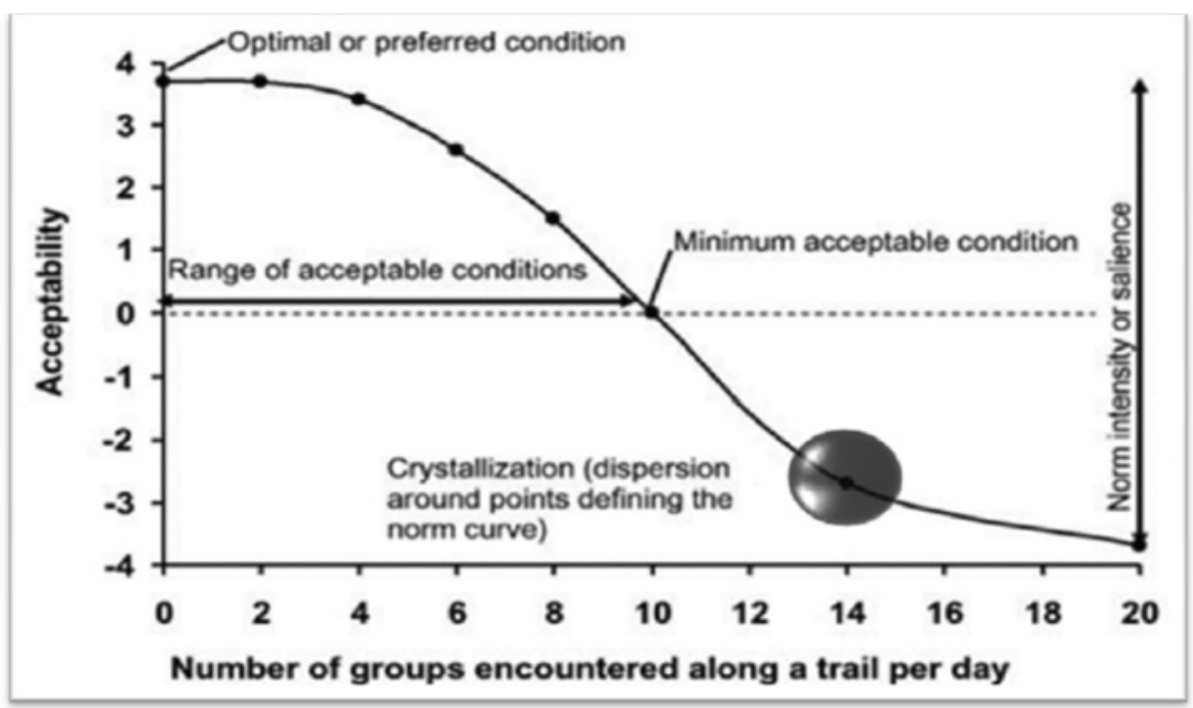

Fuente: Alazaizeh et al. (2015:3), adaptada de Manning (2007:43).

Estas normas pueden ser estudiadas usando el RPM pidiendo a los visitantes que realicen su evaluación sobre diferentes condiciones del parque. Posteriormente, se puede dibujar en un gráfico una curva de normas sociales de las puntuaciones de aceptabilidad media (eje $\mathrm{X}$ ) en función del rango de condición evaluado (eje Y). La teoría normativa es, así, una garantía para desarrollar estándares y evaluar diferentes condiciones sociales, ambientales y/o de gestión en parques nacionales y áreas afines (Alazaizeh et al., 2015).

\section{3. ÁREA DE ESTUDIO: EL CENOTE DZONBAKAL EN EL EJIDO SAN ANTO- NIO MULIX, YUCATÁN, MÉXICO}

Los cenotes son ecosistemas característicos de la península de Yucatán, comparten rasgos únicos que permiten el desarrollo y sustento de la biodiversidad y de los asentamientos humanos circundantes. La palabra cenote se castellanizó del vocablo maya "ts'onot" que significa "caverna con depósito de agua" (Beddows et al., 2007). Este término abarca toda manifestación kárstica que alcance el nivel freático, es decir cualquier espacio subterráneo 
con agua con la condición de contar con una abertura al exterior sin importar su diámetro (Gaona-Vizcayno et al., 1980).

Estos sistemas son de importancia social, cultural, ecológica y económica. Históricamente los gobernantes mayas reconocían la importancia estratégica que los cenotes representaban al permitir el acceso directo al agua subterránea, influyendo, de esta manera, en el desarrollo de sus asentamientos, además del significado espiritual que se les otorgaban (Martos, 2007). Además, los cenotes son sitios de numerosos endemismos, como la anguila ciega, Ophisternon infernale, y la dama blanca, Ogilbia pearsei, y crustáceos como Creaseria morleyi y Typhlatya campecheae (Iliffe, 1992; Reddell, 1977 y NavarroMendoza y Valdés-Casillas, 1990). En cuanto a la vegetación circundante prevalece la selva mediana subperennifolia, predominante de la región de Yucatán, aunque suele asociarse al álamo, Ficus cotinifolia (Reddell, 1981). Por otra parte, la constitución geológica calcárea de la península es causa de que el agua difícilmente se conserve en la superficie y se filtre hasta los mantos freáticos que suelen ser profundos. Por ello, los cenotes fueron para los antiguos mayas de Yucatán fuente primordial de agua y de vida (Martos, 2007).

Para algunas comunidades, el simbolismo espiritual de estos cuerpos de agua está cambiando y se hace más notable el valor económico por el rol que juegan en el desarrollo del turismo rural (Martos, 2007). Tal es el caso de San Antonio Mulix, comisaría perteneciente al municipio de Umán en el Estado de Yucatán que, según el mapa del POETY (Programa de Ordenamiento Ecológico del Territorio del Estado de Yucatán), se encuentra en territorio apto para realizar actividades enfocadas al turismo de bajo impacto. Se localiza a 49 km. de Mérida (Fig. 3), capital del Estado de Yucatán y cuenta con dos cenotes que se usan para fines turístico-recreativos: Dzombakal y Xbatún (Fig. 2). Ambos son operados por la Sociedad Cooperativa Tumben Zazil Kin Dzonot, administrada por diez habitantes del ejido: 3 de ellos mujeres y 7 hombres (Herrera, C. J. C., 2013). La ruta de más fácil acceso desde Mérida es mediante la carretera federal Muna-Uxmal. No existe transporte público hasta la entrada de los cenotes, por lo que la mayoría de los turistas llegan a bordo de autos particulares (SEDUMA, s.f.).

\section{Figura 2 \\ CENOTES DZOMBAKAL Y XBATÚN EN SAN ANTONIO MULIX}
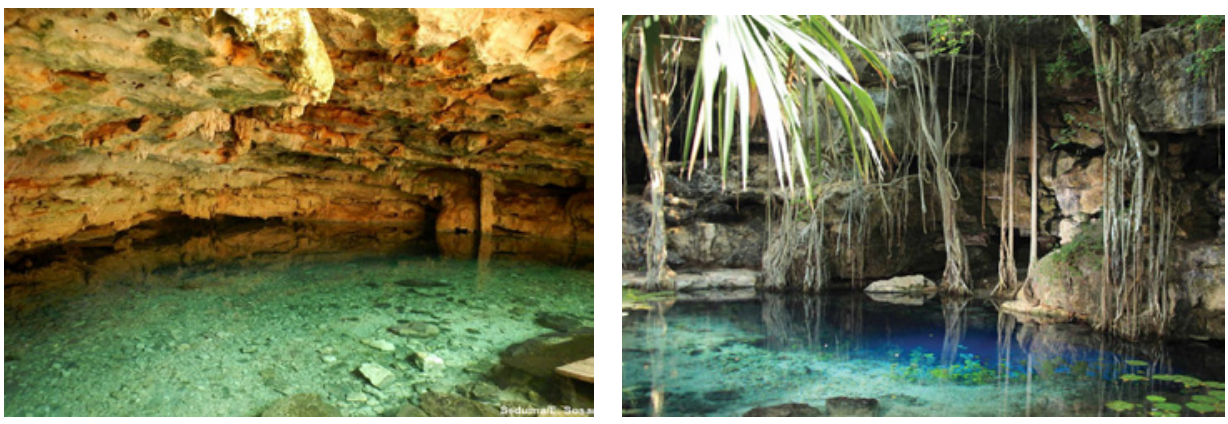


\section{Figura 3 \\ LOCALIZACIÓN DEL EJIDO SAN ANTONIO MULIX}

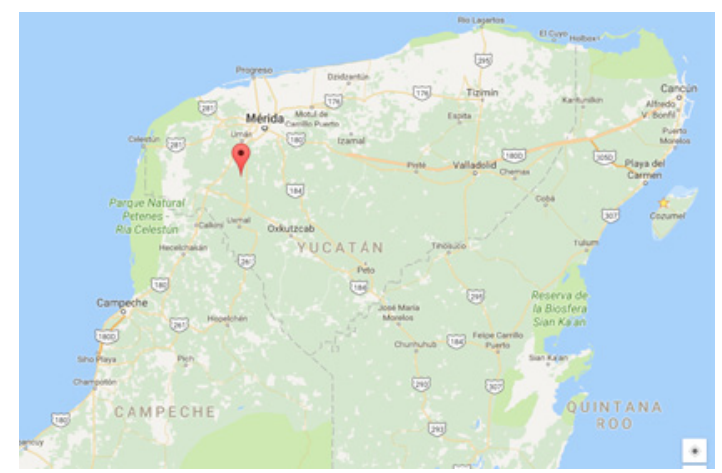

Fuente: Google Maps.

En términos de infraestructura, el Ejido cuenta con cabañas para poder pernoctar y un restaurante para poder almorzar y cenar. Adicionalmente, la cooperativa ofrece servicio de renta de bicicletas, equipo de snorkel y campismo. Sin embargo, los principales atractivos son, sin duda, sus cenotes Dzonbakal y X’Batún, que se localizan a un par de kilómetros de la entrada del Ejido.

Dzombakal, se caracteriza por ser un cenote semi-abierto de caída libre con un área de espejo de agua de $202 \mathrm{~m}^{2}$ (Fig. 4), cuenta con una bóveda superficial sin presencia de espeleotemas (cenoteando.org, s.f). Se pueden apreciar las raíces de Ficus cotinifolia que se introducen al agua y en lo alto de la bóveda se hayan nidos de golondrina, Hirundo rustica; el acceso es mediante una escalera de mampostería complementada de un barandal de madera rústico (SEDUMA, s.f.), además cuenta con un área de recreación de $18 \mathrm{~m}^{2}$ lo que suma un área total de $220 \mathrm{~m}^{2}$ para el uso turístico-recreativo, ofreciendo al turista la opción del baño y el servicio de vestuarios.

\section{Figura 4 \\ ÁREA DE LA SUPERFICIE DEL ESPEJO DE AGUA DEL CENOTE DZOMBAKAL}

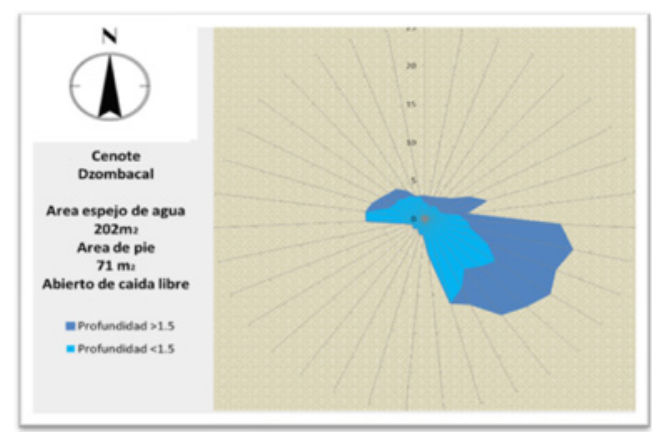

Fuente: cenoteando.org, s.f. 
En los últimos años, San Antonio Mulix y sus dos cenotes han experimentado un aumento sustancial de sus turistas provenientes, principalmente de la ciudad de Mérida (ver Fig. 5). En el año 2015 los cenotes recibieron 41,484 turistas, y para el año 2016 se registraron un total de 46,767, principalmente de nacionalidad mexicana en ambos años, lo que representa un crecimiento de $12.7 \%$ en la tasa de visitantes.

\section{Figura 5}

\section{PROMEDIO MENSUAL DE TURISTAS EN 2015 Y 2016 VISITANDO LOS CENOTES DEL EJIDO DE SAN ANTONIO MULIX, YUCATÁN}

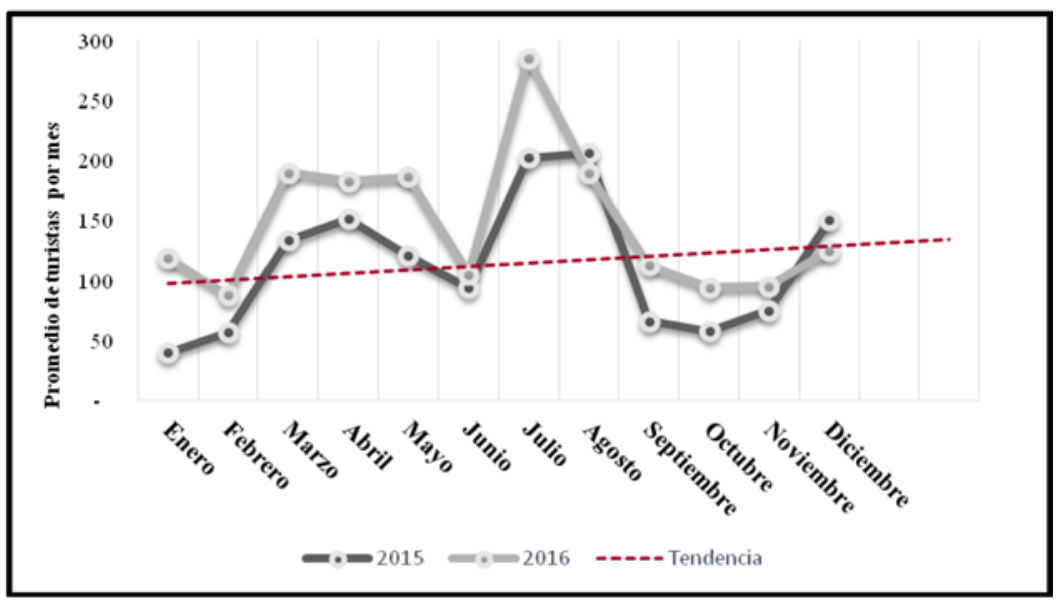

Fuente: Elaboración propia a partir del libro de visitas del Ejido San Antonio Mulix, Yucatán.

\section{METODOLOGÍA}

Se han utilizado dos enfoques para medir la capacidad de carga perceptual en áreas naturales. Por un lado, un enfoque narrativo y numérico tradicional (Shelby y Heberlein, 1986), y, por otro, más posterior, un enfoque visual (Manning, Lime, Freimund y Pitt, 1996). Ambos enfoques se basan en pedir a los visitantes y/o turistas que evalúen la aceptabilidad sobre el número de visitantes dispuestos a ver al mismo tiempo en un sitio determinado. Sin embargo, la forma de presentar esta condición de saturación en un cuestionario, difiere. El enfoque narrativo y numérico (tradicional) utiliza una descripción de las condiciones, mientras que en el enfoque visual se utilizan fotografías manipuladas por ordenador para reflejar esa gama de condiciones.

Los dos enfoques de medición se consideran válidos, pero en algunas situaciones específicas, algunas técnicas pueden ser más adecuadas (Manning, 1999). Por ejemplo, en la investigación relacionada con la saturación o multitud, como es el caso del cenote de Dzombakal que aquí se presenta, el enfoque visual puede ser más apropiado ya que los niveles de uso en el sitio son relativamente altos y complejos (Manning y Krymkowski, 2010; Manning, 1999). 
Así, para este estudio se optó por utilizar el enfoque visual y se manipuló con el programa Photoshop una fotografía del cenote Dzombacal, de manera que se obtuvieran 6 láminas conteniendo diferente número de turistas cada una de ellas (ver fig. 6). La primera lámina (fotografía original) tenía cero visitantes y la última tenía el número de visitantes que la capacidad de carga física del cenote arrojó.

La capacidad de carga física (CCF) se obtuvo midiendo la superficie visitable del cenote, es decir, el espejo de agua $\left(202 \mathrm{~m}^{2}\right)$ más toda el área localizada alrededor del cenote y donde el turista puede estar de pie o sentado descansando $\left(18 \mathrm{~m}^{2}\right)$, dando como resultado un total de $220 \mathrm{~m}^{2}$. Y después, se dividió la superficie visitable entre $4 \mathrm{~m}^{2}$, que es área vital utilizada en estudios similares (García Hernández, 2001), dando como resultado 55 personas.

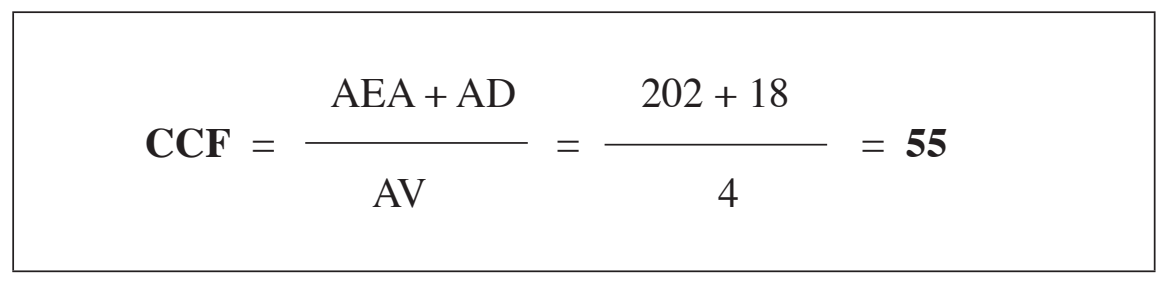

CCF (Capacidad de Carga Física $)=55$

$\mathrm{SV}$ (Superficie Visitable) $=220 \mathrm{~m}^{2}$

AEA (Área de Espejo de Agua) $=202 \mathrm{~m}^{2}$

$\mathrm{AD}($ Área de Descanso $)=18 \mathrm{~m}^{2}$

$\mathrm{AV}($ Área Vital $)=4 \mathrm{~m}^{2}$

La capacidad de carga física de 55 personas en el cenote de Dzombacal representa la cantidad de personas que se incluyeron en la fotografía número 6, representando el número máximo de turistas que puede albergar el cenote. De esta manera, la fotografía número 1 tenía 0 personas, la número 2, 11 personas y así aumentaba la cantidad de personas en intervalos de 11 hasta llegar a la fotografía 6 que contenía 55 personas.

Se utilizó un muestreo no probabilístico por conveniencia y se determinó el tamaño de la muestra por fórmula estadística, dando como resultado una muestra de 191 turistas La muestra es representativa de la población total de turistas que han visitado el Ejido durante el año 2016, obteniendo el total de visitantes por mes que quedan registrados en el libro de visitas de San Antonio Mulix.

La encuesta fue realizada durante el mes de abril de 2017 y se obtuvo una tasa de respuestas del $65 \%$ (124 turistas/191 turistas). De las 124 personas que respondieron a la encuesta, 51 fueron residentes locales, es decir, del estado de Yucatán, principalmente de Mérida y los municipios localizados alrededor de San Antonio Mulix. Participaron también 51 turistas nacionales, considerados como todos los residentes de otros estados de México diferentes a Yucatán; y 22 turistas extranjeros. A cada turista se le mostraron las 6 fotografías, una por una, y se le pidió que las evaluaran en un escala del 1 al 9, siendo 1 el nivel de aceptación más bajo y 9 el nivel más alto. Asimismo se le pidió expresar la 
cantidad más alta que estaría dispuesto a pagar en cada una de las imágenes. La intención de esta pregunta era medir la disposición del turista a pagar más por sitios menos saturados. La distinción entre visitantes locales, nacionales y extranjeros se hizo para identificar si existen diferencias en el nivel de aceptación en función del lugar de residencia.

\section{Figura 6 \\ LAS 6 FOTOGRAFÍAS UTILIZADAS EN EL ESTUDIO}

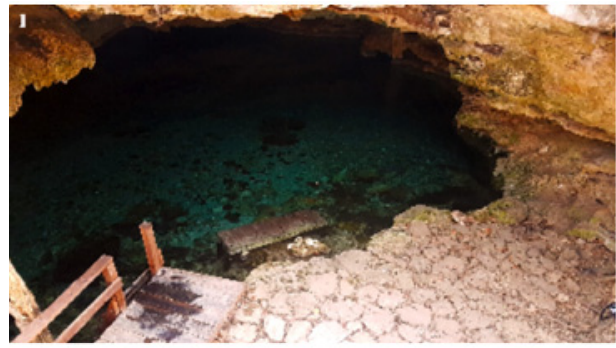

Fotografia 1:0

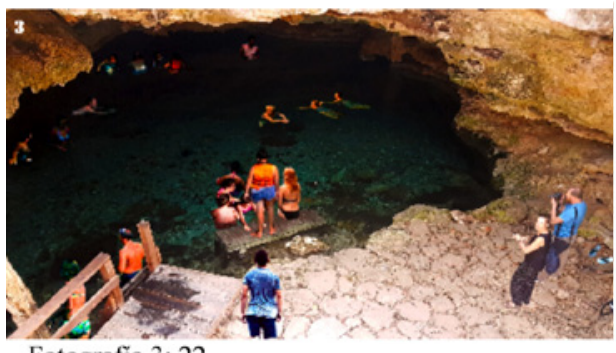

Fotografia 3: 22

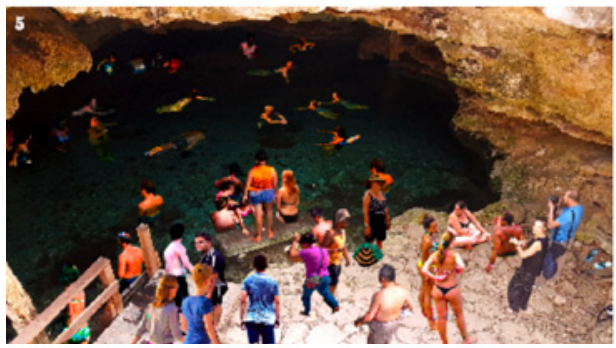

Fotografia 5: 44

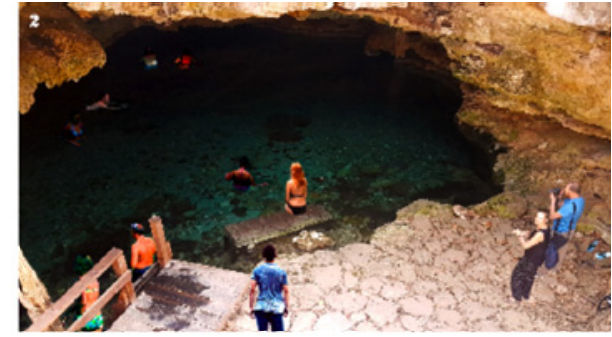

Fotografia 2: 11

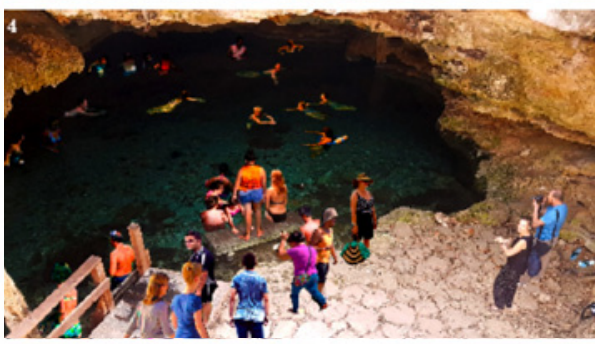

Fotografia $4: 33$

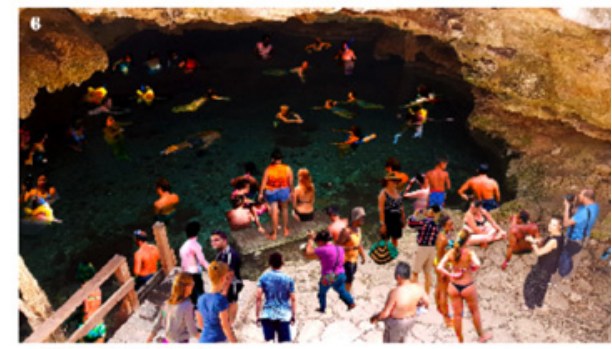

Fotografía 6: 55

Fuente: Elaboración propia

\section{RESULTADOS PRINCIPALES}

Los hallazgos permitieron establecer estándares de capacidad de carga psico-social o perceptual (tolerancia psicológica individual ante la presencia de un conjunto social de visitantes o turistas en un lugar de interés turístico) para cenotes y confirman que el lugar de residencia influye en los niveles de aceptación de multitud que tiene el turista. Los 
turistas locales (residentes en la ciudad de Mérida y municipios de alrededor) mostraron una mayor tolerancia a la saturación de visitantes, siendo 40 el número de personas a partir del cual su nivel de aceptación comienza a disminuir (Fig. 7). En el caso de los turistas nacionales, su nivel de aceptación comienza a decrecer con 33 personas concentradas al mismo tiempo en el cenote (Fig. 8). Los turistas extranjeros mostraron la tolerancia más baja a la saturación, siendo 23 el número máximo de personas a partir del cual, el cenote ya no les resulta aceptable (Fig. 9). Así, es evidente que los locales toleran poco menos del doble del número de personas que los extranjeros y, a diferencia de los nacionales y los extranjeros, un cenote vacío no es el que les produce el nivel de aceptación más alto, sino aquel que tiene 11 personas, comenzando a disminuir a partir de este número.

Figura 7

NIVEL DE ACEPTACIÓN DE TURISTAS LOCALES

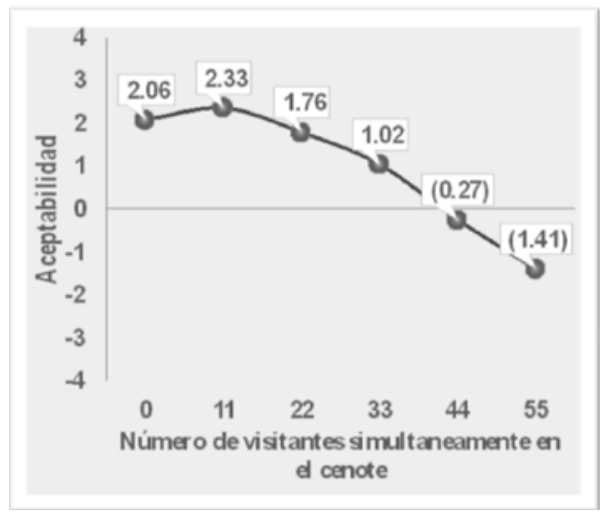

Fuente: Elaboración propia.

\section{Figura 8 \\ NIVEL DE ACEPTACIÓN DE TURISTAS NACIONALES}

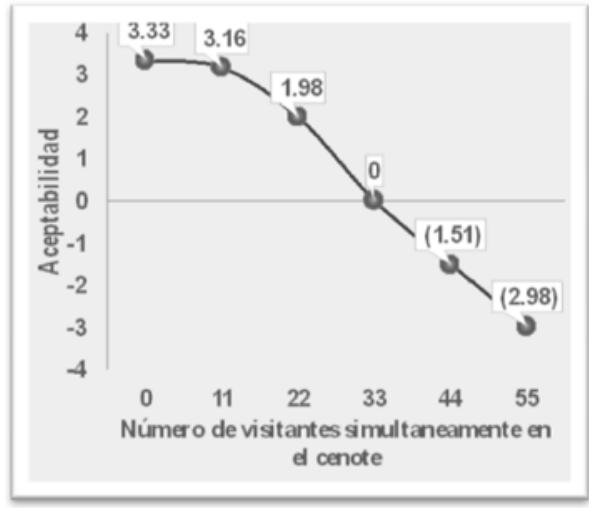

Fuente: Elaboración propia. 


\section{Figura 9 \\ NIVEL DE ACEPTACIÓN DE TURISTAS EXTRANJEROS}

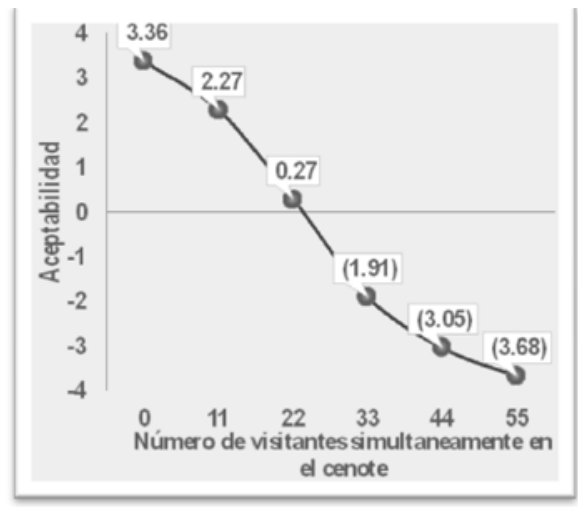

Fuente: Elaboración propia.

Por otro lado, los resultados de evaluar la disposición a pagar más por sitios menos saturados arrojaron que, en general, el turista está dispuesto a pagar más por estar en un cenote vacío o con pocas personas. Se identificaron diferencias entre los residentes locales, nacionales y extranjeros. Los locales resultaron ser los menos dispuestos a pagar más por sitios menos saturados con una diferencia de 23.63 pesos mexicanos entre el máximo dispuesto a pagar por un cenote vacío (58.22 pesos) y el mínimo dispuesto a pagar por un cenote saturado (34.59 pesos) (Fig. 10). En el caso de los nacionales, la diferencia entre la cantidad máxima (83.04 pesos) y la cantidad mínima dispuestos a pagar (9.37 pesos) fue de 73.67 pesos (Fig. 11). Los extranjeros presentaron la disposición más alta a pagar por sitios vacíos o poco saturados, con una diferencia de 126.52 pesos entre la cantidad máxima a pagar por un cenote vacío (129.09 pesos) y la cantidad mínima a pagar por un cenote saturado (2.50 pesos) (Fig. 12).

\section{Figura 10}

\section{CANTIDAD DISPUESTA A PAGAR EN TURISTA LOCALES}

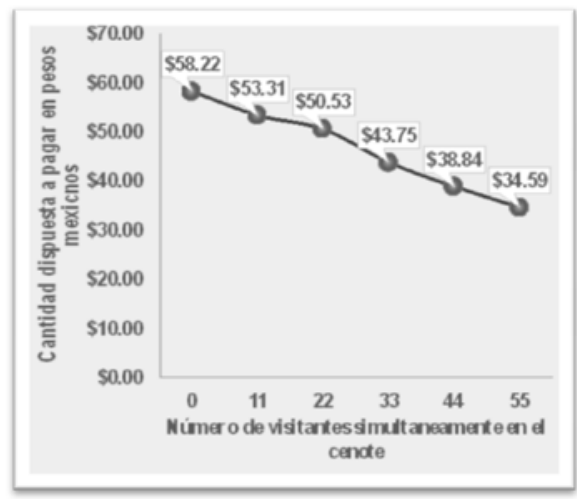

Fuente: Elaboración propia. 


\section{Figura 11}

\section{CANTIDAD DISPUESTA A PAGAR EN TURISTAS NACIONALES}

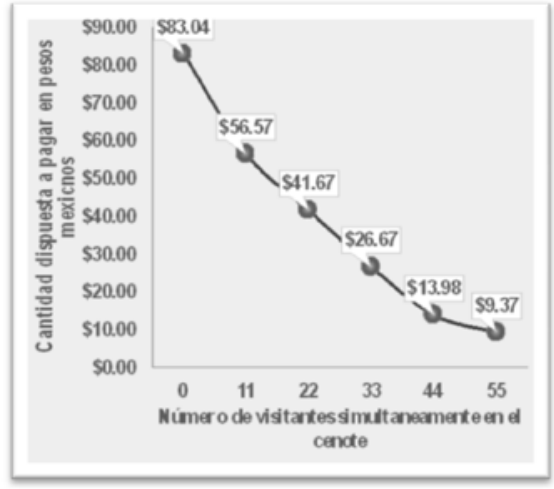

Fuente: Elaboración propia.

Figura 12

\section{CANTIDAD DISPUESTA A PAGAR EN TURISTAS EXTRANJEROS}

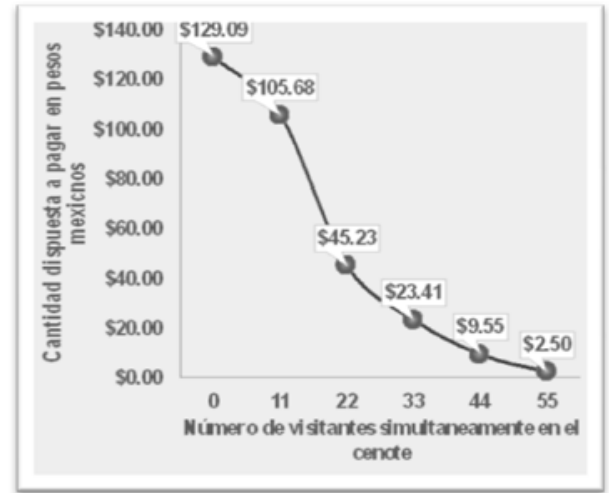

Fuente: Elaboración propia.

\section{DISCUSIÓN}

Llegados a este punto, lo que ahora nos planteamos son las directrices a tomar por los gestores de los cenotes para que esto influya positivamente en el turismo de la zona y también en la sostenibilidad del mismo para su continuidad como fuente de recursos turísticos y económicos.

En nuestra opinión, y basándonos en los primeros resultados obtenidos con el trabajo de campo, la cooperativa que gestiona el cenote debe establecer un límite en la entrada de personas permaneciendo simultáneamente en el mismo, pues ya hemos visto que el nivel de aceptabilidad disminuye cuantas más personas haya a la vez en el cenote, con lo cual, 
no sólo es una cuestión de afección negativa al medio ambiente del lugar sino que también afecta al nivel de carga perceptual del visitante o turista, que se refiere a una dimensión psicosocial que está relacionada con la calidad de la experiencia de todas aquellas personas que se acercan a conocer un determinado lugar. Por lo tanto, si el visitante no tolera el alto número de personas que hay en el momento de su ingreso al cenote, existen altas probabilidades de que no vuelva o, incluso, manifieste su experiencia negativa a familiares, amigos o terceras personas.

Por otra parte, y teniendo en cuenta, tanto el nivel de ingresos como el origen de los diferentes visitantes, creemos, a la luz del estudio realizado, que el precio de la entrada al cenote debe variar en función del origen o procedencia del visitante; teniendo la ventaja de pagar una cantidad menor aquellas personas que pertenecen al municipio o son de nacionalidad mexicana, $y$, por otra parte, una cantidad superior a pagar para los visitantes o turistas extranjeros, pues así se estipula la visita a monumentos, museos y otros lugares de interés turístico en otros países, teniendo una ventaja añadida todas aquellas personas que poseen la nacionalidad del país de destino. En este sentido, son, precisamente, los turistas extranjeros, atendiendo a los datos obtenidos, los que estarían dispuestos a abonar una cantidad de dinero mayor por el precio de la entrada por visitar el cenote y encontrar un número pequeño de personas en el lugar, seguidos de los turistas nacionales y teniendo en cuenta que para este tipo de turistas o visitantes su nivel de renta es mayor que si lo comparamos con el nivel de renta de los visitantes locales, en los cuales no se manifiesta una gran diferencia en cuanto a la cantidad a abonar por la entrada, puesto que para ellos no es tan significativo el hecho de que el cenote esté vacío, semivacío o completamente lleno, como se ha podido observar en los resultados de la encuesta (ver Figura 10).

\section{CONCLUSIONES}

Una de las principales conclusiones que se han obtenido de los resultados de este estudio, referido al cenote Dzombakal, perteneciente al ejido de San Antonio Mulix, en el estado de Yucatán, es que se puede afirmar que hay una relación inversamente proporcional entre el número de visitantes y el nivel de aceptabilidad, es decir, a mayor confluencia de personas al mismo tiempo en el cenote se denota un decremento del nivel de aceptabilidad por parte de los visitantes y/o turistas que aumenta progresivamente según la procedencia geográfica de los mismos, siendo menor en los visitantes extranjeros, seguidos de los nacionales y, por último, los locales que son el tipo de visitantes que presentan una mayor tolerancia o aceptación a un gran número de personas visitando simultáneamente el cenote.

En adición, se puede concluir que, en el caso de los visitantes locales, se encontró que un cenote vacío no presentaba el nivel de aceptabilidad más alto, como, en cambio, sí fue el caso de los visitantes nacionales y extranjeros, con mayor proporción en éstos últimos.

Por otra parte, el nivel de aceptación en el número de visitantes simultáneamente en un cenote varía en función del origen del turista: llegando hasta 40 el número de visitantes aceptable para los visitantes locales, hasta 33 para los nacionales y hasta 23 para los visitantes extranjeros. 
Además, y teniendo en cuenta el aspecto económico con respecto a la cantidad monetaria que el visitante estaría dispuesto a pagar como precio de entrada al lugar, hay que señalar que en los tres tipos de procedencia de los visitantes encuestados (locales, nacionales y extranjeros), se observa una relación inversamente proporcional entre la cantidad dispuesta a pagar y el número de turistas que se le muestran en las fotos, teniendo ésta un mayor impacto en el caso de los visitantes nacionales y extranjeros, existiendo una variación entre cantidad máxima y mínima dispuesta a pagar de \$73.67 y \$126.52 respectivamente. En contraste, hay que apuntar que los visitantes locales son menos sensibles a la cantidad de personas con respecto a su disposición a pagar, pues la diferencia entre el máximo y mínimo que ellos pagarían se situaría en tan sólo \$23.63, independientemente del número de personas que encontraran simultáneamente en su visita al cenote.

En definitiva, se presenta una constante en los tres tipos de visitantes encuestados, ya que, cuanto menos visitantes encuentran, mayor es su disposición a pagar, llegando a pagar los locales un máximo de $\$ 58.22$ y un mínimo de $\$ 34.59$ por el precio de una entrada; los nacionales un máximo de $\$ 83.04$ y un mínimo de $\$ 9.37$; y, por último, los extranjeros que abonarían por la entrada hasta un máximo de \$129.09 y un mínimo de \$2.50.

\section{BIBLIOGRAFÍA}

ALAZAIZEH, M.M., HALLO, J.C., BACKMAN, S.J., NORMAN, W.C. y VOGEL, M. A. (2015): «Crowding standards at Petra Archaeological Park: a comparative study of McKercher>s five types of heritage tourists», Journal of Heritage Tourism, vol. 11, $\mathrm{n}^{\circ}$ 4, pp. 364-381.

ÁLVAREZ, M.P. (2010): «Evaluación de la capacidad de carga. Una herramienta para el manejo y la conservación de los sitios patrimoniales», Canto Rodado: Revista especializada en patrimonio, $\mathrm{n}^{\circ} 5$, pp. 221-247.

AMADOR, K., GINO, R., ARROYO, L. y SERRANO, R.C. (2013): «La dimensión social en la capacidad de carga turística: estudio del caso playa Chen Río, Isla de Cozumel, México»,Terr@Plural,vol.7,nº 1,pp.157-170. Doi: 10.5212/TerraPlural.v.7i1.0010

BEDDOWS, P., BLANCHON, P., ESCOBAR, E. y TORRES-TALAMANTE, O. (2007): «Los cenotes de la península de Yucatán», Arqueología Mexicana, n 83, pp. 32-35.

BURNS, R. C., ARNBERGER, A. y VON RUSCHKOWSKI, E. (2010): «Social Carrying Capacity Challenges in Parks, Forests , and Protected Areas: An Examination of Transatlantic Methodologies and Practices», International Journal of Sociology, vol. $40, \mathrm{n}^{\mathrm{o}} 3$, pp. 30-50.

Cenoteando.org, (s.f.): Página web en desarrollo creada por estudiantes de la Licenciatura en Manejo Sustentable de Zonas Costeras de la UNAM, Sisal, Yucatán (México). Recuperado de www.cenoteando.org.

COCCOSSIS, H. (2013): "Carrying Capacity as a tool for the Management of Tourism Pressures on Heritage Sites», in N. Agnew and M. Demas (Eds.): Visitor Management and Carrying Capacity at World Heritage Sites in China. Mogao Grottoes, China: The Getty Conservation Institute, pp. 35-38. 
CÓRDOBA, J. (1984): Descentralización, territorio y ambiente en la Península de Yucatán: una mirada geográfica desde el enfoque de centralidad. Mérida, México, CINVESTAV.

DONNELLY, M.P., VASKE, J. y SHELBY, B. (1992): «Establishing management standards: Selected examples of the normative approach», in B. Shelby, G. Stankey and B. Shindler (Eds.): Defining wilderness quality: The role of standards in wilderness management. A workshop proceedings (USDA Forest Service General Technical Report PNW-305).

DRUM, A. y MOORE, A. (2002): Desarrollo del ecoturismo: Un manual para los profesionales de la conservación. Arlington, Virginia (USA), The Nature Conservancy.

ÉQUIPE MIT. (2002): Tourism 1. Lieux communs. París, Éditions Belin.

GAONA-VIZCAYNO, S., GORDILLO DE ANDA, T. y VILLASUSO-PINO, M. (1980): «Cenotes, karst característico: mecanismos de formación», Rev. Univ. Nac. Autón. México, vol. 4, $n^{\circ}$ 3, pp. 32-36.

GARCÍA HERNÁNDEZ, M., DE LA CALLE VAQUERO, M. y MÍNGUEZ GARCÍA, M.C. (2011): «Capacidad de carga turística y espacios patrimoniales. Aproximación a la estimación de la capacidad de carga del conjunto arqueológico de Carmona (Sevilla, España)», Boletín de La Asociación de Geógrafos Españoles, n 57, pp. 219-241.

HERRERA, C.J.C. (2013): Explaining autonomy in community-based ecotourism: A comparative study of two proyects in Yucatan, Mexico. Tesis de Grado no publicada del Departamento de Geografía, Universidad de Londres. King's College London. MA Degree in Tourism, Environment and Development. Londres, Inglaterra.

ILIFFE, T.M. (1992): «An annotated list of the troglobitic anchialine and freshwater fauna of Quintana Roo», in Diversidad Biológica en la Reserva de la Biosfera de Sian Ka'an, Quintana Roo, México. CICRO/SEDESOL, Chetumal, vol. II, pp. 197-217.

JACKSON, J. (1965): «Structural characteristics of norms», in I. D. Steiner and M. Fishbein (Eds.), Current studies in social psychology. New York, NY: Holy, Rinehart and Winston, pp. 301-309.

JACKSON, J. (1966): «A conceptual and measurement model for norms and roles», The Pacific Sociological Review, vol. 9, $\mathrm{n}^{\circ}$ 1, pp. 35-47.

MANNING, R.E. (1999): «Crowding and carrying capacity in outdoor recreation from normative standards to standards of quality», in E. L. Jackson and T. L. Burton (Eds.): Leisure studies: Prospects for the twenty-first century. State College, PA: Venture Publishing, pp. 323-334.

MANNING, R.E. y ANDERSON, L.E. (2012): Managing outdoors recreation: Case Studies in the National Parks. Wallingford, UK, CABI Publishing.

MANNING, R.E. y KRYMKOWSKI, D.H. (2010): «Standards of quality for parks and protected areas», International Journal of Sociology, vol. 40, n 3, pp. 11-29.

MANNING, R.E., LIME, D., FREIMUND, W. y PITT, D. (1996): «Crowding norms at frontcountry sites: A visual approach to setting standards of quality», Journal of Leisure Sciences, vol. 18, $\mathrm{n}^{\circ} 1$, pp. 39-59.

MANNING, R.E., VALLIERE, G.A. y WANG, B. (1999): «Crowding Norms: Alternative Measurement Approaches», Journal of Leisure Sciencies, vol. 21, nº 2, pp. 97-115. 
MARÍN GUARDADO, G., GARCÍA DE FUENTES, A. y DALTABUIT GODÁS, M. (Coords.) (2012): Turismo, globalización y sociedades locales en la Península de Yucatán, México. La Laguna (Tenerife): PASOS, RTPC. www.pasososnline.org. Colección PASOS Edita ${ }^{\circ} 7$.

MARTOS, L. (2007): «Los cenotes en la actualidad, entre la veneración y la explotación», Arqueología Mexicana, $\mathrm{n}^{\circ}$ 83, pp. 32-35.

NAVARRO-MENDOZA, M. y VALDÉS-CASILLAS, C. (1990): «Peces cavernícolas de la Peninsula de Yucatan en peligro de extinción, con nuevos registros para Quintana Roo», en J.L. Camarillo y F. Rivera (Comps.): Áreas naturales protegidas en México y especies en extinción. México: ENEP-Iztacala, UNAM, pp. 218-241.

REDDELL, J.A. (1977): «A preliminary survey of the caves of the Yucatan Peninsula», Assoc. Mex. Cave. Stud. Bull., nº 6, pp. 215-296.

REDDELL, J.A. (1981): «A review of the cavernicole fauna of Mexico, Guatemala, and Belize», Texas Mem. Mus. Bull, no 27, pp. 1-327.

SEDUMA (s.f.): CENOTES: Ficha Técnica. Recuperado el 5 de agosto de 2017, de http://www.seduma.yucatan.gob.mx/cenotes-grutas/fichas-tecnicas/Yucatan_Uman_ cenote_Dzonbacal.pdf

SHELBY, B. y HEBERLEIN, T.A. (1986): Carrying capacity in recreation settings. Corvallis, Oregon State: University Press.

SHELBY, B. y VASKE, J.J. (1991): «Using normative data to develop evaluative standards for resource management: A comment on three recent papers», Journal of Leisure Research, vol. 23, n 2, pp. 173-187.

SHELBY, B., VASKE, J.J. y HARRIS, R. (1988): «User standards for ecological impacts at wilderness campsites», Journal of Leisure Research, vol. 20, n 3, , pp. 245-256.

SCHÉOU B. (2009): Du tourisme durable au tourisme équitable: Quelle éthique pour le tourisme de demain? París, De Boeck Supérieur.

TUDELA SERRANO, M.L. y GIMÉNEZ ALARTE, A.I. (2008): «Determinación de la capacidad de carga turística en tres senderos de pequeño recorrido en el municipio de Cehegín (Murcia)», Cuadernos de Turismo, n 22, pp. 211-229.

VASKE, J. J. y DONNELLY, M.P. (1988): «Normative evaluations of wildlife management». Paper presented at the Annual Congress of the National Recreation and Park Association, Indianapolis, Indiana.

VASKE, J., GRAEFE, A., SHELBY, B. y HEBERLEIN, T. (1986): «Backcountry encounter norms: Theory, method, and empirical evidence», Journal of Leisure Research, $\mathrm{n}^{\circ}$ 18, pp.137-153.

VASKE, J.J. y WHITTAKER, D. (2004): «Normative approaches to natural resources», en M.J. Manfredo, J.J. Vaske, B.L. Bruyere y D.R. Field (Eds.): Society and natural resources: A summary of knowledge. Jefferson, MO, Modern Litho, pp. 283-294. 